\section{Thin-Film $\mathrm{LiNbO}_{3}$ Pyroelectric Detectors Coated with Multiwalled CNTs Enhance Sensitivity}

The current generated by a pyroelectric detector is inversely proportional to its thermal mass. Therefore, a practical limit for building more sensitive pyroelectric detectors is imposed by conventional lapping and polishing of the detector plate. Thin-film pyroelectric detectors fabricated by crystal ion slicing show higher sensitivity than those prepared by conventional lapping and polishing while maintaining the pyroelectric properties of the bulk material. By coating the detectors with a low mass thermal absorber, such as carbon nanotubes (CNTs), the sensitivity of the devices can be further enhanced, with a uniform spectral responsivity. J.H. Lehman and K.E. Hurst from the National Institute of Standards and Technology, Colorado, A.M. Radojevic from The Charles Stark Draper Laboratory, Mass., A.C. Dillon from National Renewable Energy Laboratory, Colorado, and R.M. Osgood Jr. from Columbia University, New York, reported such improvements in this type of pyroelectric detector when compared to a nickel-coated detector, in the April 1 issue of Optics Letters (p. 772).

The detector consisted of a 10- $\mu$ m thick freestanding film of $\mathrm{LiNbO}_{3}$, fabricated by crystal ion slicing by bombardment of the parent material with high-energy helium ions. The detector film is separated by acid etch or thermal shock at a depth where the ions run out of momentum. The film is then coated with $250-\mathrm{nm}$ thick nickel electrodes on each face and packaged as a freestanding detector. The researchers coated the freestanding detector with a 5-10- $\mu$ m thick layer of commercially available multiwalled CNTs (MWNTs) by spraying it with a dispersion of $0.33 \mathrm{~g}$ of these nanotubes in $13.4 \mathrm{ml}$ of chloroform by an airbush technique. MWNTs were chosen over singlewall CNTs (SWNTs) to avoid the spectral features characteristic of interband transi- tions of metallic and semiconducting materials, which SWNTs readily exhibit.

The MWNT-coated detector, black due to the optical properties of the individual tubes and the topology of the bulk, appeared like "a mat of bundled ropes with various clumps interspersed" under the scanning electron microscope. The clumps are believed to be catalyst metals and non-nanotube carbons. The MWNT coating is attached to the electrode surface by van der Waals forces and by interactions with pi electrons in the orbital perpendicular to the axis of the tubes. The coating is robust: it can be scratched with a rigid stylus, but it is not removed by forced air or water. The increase in spectral responsivity of the MWNT-coated detector is about four times over the spectral region from $600 \mathrm{~nm}$ to $1800 \mathrm{~nm}$ compared to a $\mathrm{Ni}$-coated detector, with minimal penalty to the frequency response. According to the researchers, the enhanced performance, along with the facile and inexpensive application of the coating, is immediately achievable and is promising for other types of radiometric detectors and thermal detector arrays.

JOAN J. CARVAJAL

\section{Superconductivity and Relativity Meet in a Monolayer of Graphene}

Until recently, superconductivity and the theory of relativity had very little to do with each other. However, H. Heersche, P. Jarillo-Herrero, and their colleagues at Delft University of Technology's Kavli Institute for Nanoscience and the FOM Foundation have detected superconducting properties in a material "in which charge carriers behave as massless chiral relativistic particles." Their device — which consists of graphene attached to superconductors-also functions as a bipolar transistor for superconducting currents.

As reported in the March 1 issue of Nature (p. 56; DOI: 10.1038/nature05555) the researchers attached graphene to oxi- dized silicon substrates by mechanical exfoliation. They inspected the samples with an optical microscope to determine the thinnest graphene flakes, and then used electron beam lithography to fabricate metal contacts. The superconducting contacts are formed from a $\mathrm{Ti} / \mathrm{Al}$ bilayer (10/70 nm thick, see Figure 1). In a superconductor, the electrical resistance completely disappears at very low temperatures. This means that an electrical current can continue to flow without a voltage being applied, known as a supercurrent. When graphene-which itself has no superconducting properties-is joined together with a superconductor, it can behave like a superconductor. This effect has been identified in many other nonsuperconducting materials and is known as the Josephson-effect. The researchers at Kavli Institute were able to measure the relativistic Josephson effect. They observed the quantum Hall effect (QHE) and demonstrated that the QHE can be used to identify single-layer devices even in mesoscopic samples.

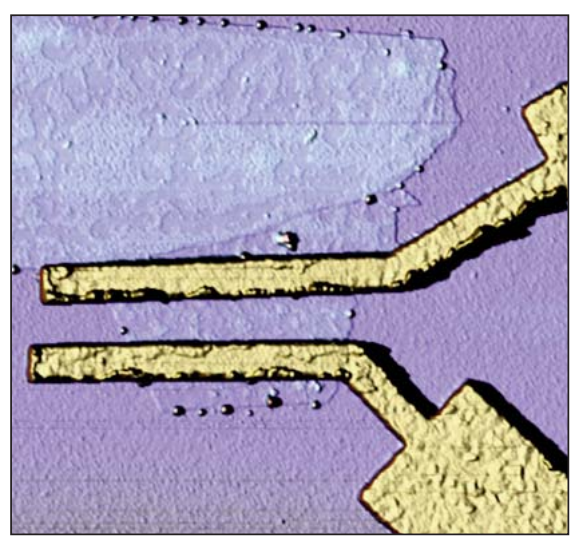

Figure 1. Image of a layer of graphene attached to superconductors, captured with an atomic force microscope. The distance between each of the superconductors is $300 \mathrm{~nm}$. 
The researchers have also demonstrated that graphene can serve as a supercurrent transistor. Heersche and colleagues have created a device in which the supercurrent can be regulated by a voltage, similar to the control exhibited in a conventional semiconductor transistor in which the current is controlled by a gate electrode. Graphene is not a semiconductor, but rather a semi-metal. This means that not only the size of the supercurrent can be regulated, but also the type of charge carrier, according to the researchers. This can be electron Cooper pairs (with a negative charge) or hole Cooper pairs (with a positive charge). The researchers therefore report the fabrication of a bipolar supercurrent transistor.

The researchers said that, in sweeping the position of the Fermi level from the valence band to the conduction band, they sweep through the Dirac point. They said that, even then, the supercurrent remains finite, which they argue demonstrates that the electronic transport in graphene is phase coherent, even when the Fermi level is located at the Dirac point.

The researchers also discuss the effects of time reversal symmetry in graphene in the superconducting state based upon occupation of the two different K-points in the band structure by the two Cooper electrons. They contrast this to graphene in the normal state in which transport is determined by a single-valley band structure.

\section{Photoconductive Atomic Force Microscopy Maps Photocurrent in Solar Cells}

Solar cells made from organic semiconductor blends are currently low in power conversion efficiency. Typically, bound excitons created from absorbed light must dissociate into free charges at donoracceptor interfaces. However, efficient light absorption requires a $\sim 100$-nm film thickness, but excitons can only travel $\sim 10 \mathrm{~nm}$ before they decay. This so-called exciton bottleneck can be overcome with nanostructured organic solar cells with large internal surface areas. For example, donor and acceptor materials can be processed in a common solvent to a phase-separated film. Solar cell performance has been shown to depend very much on film morphology, but conventional characterization tools either lack the resolution needed to approach the exciton diffusion length, or do not provide direct information on the correlation between structure and local per- formance. Recently, however, assistant professors D.S. Ginger and G.P. Bartholomew, along with their students D. Coffey, O. Reid, and D. Rodovsky at the Department of Chemistry, University of Washington, Seattle, have demonstrated that photoconductive atomic force microscopy (pcAFM) can map local photocurrents in polymer-fullerene blend solar cells with 20-nm resolution.

As reported in the March issue of Nano Letters (p. 738; DOI: 10.1021/nl062989e), Ginger and co-researchers applied pcAFM to poly[2-methoxy-5-(3',7'dimethyloctyl-oxy)-1,4-phenylene vinylene]:(6,6)-phenyl- $\mathrm{C}_{61}$-butyric acid methyl ester (MDMO-PPV:PCBM) — a well known photovoltaic blend. Standard, mm-sized photovoltaic cells were fabricated by spincoating and curing a 40-nm layer of PEDOT:PSS (a conductive mixture of two ionic polymers) onto glass substrates coated with indium tin oxide (ITO). An 80-nm thick film of MDMO-PPV:PCBM was then spin-coated onto the ITO from either chlorobenzene, xylene, or toluene solutions in order to obtain varying degrees of phase separation in the active layer. Aluminum top contacts 40 -nm thick were evaporated through a shadow mask, thereby defining
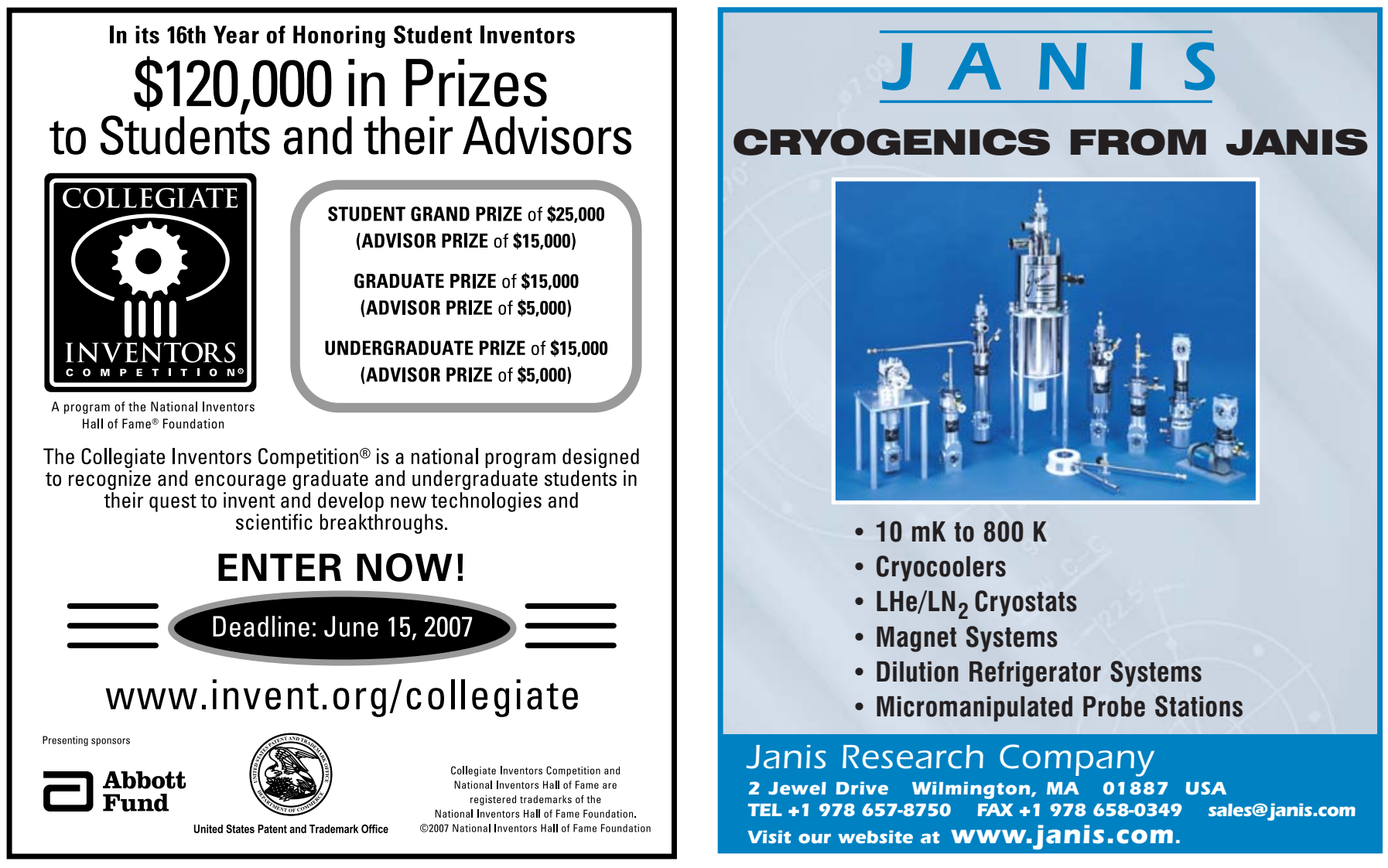